\title{
Corpora de inglês aeronáutico: desafios para o estudo da área e proposta de trabalho conjunto
}

\section{Aeronautical English Corpora: challenges to the study of the field and proposal of joint collaboration}

"É preciso reforçar o intercâmbio entre esses campos de atuação [línguas estrangeiras e corpora.] Tal troca, no entanto, deve ocorrer em uma via de mão dupla: a pesquisa deve informar o fazer docente da mesma forma que o trabalho do professor deve prover parâmetros para a realização de novas investigações."

Viana, V. e Tagnin, S. 2011: 23

Patrícia Tosqui-Lucks Malila Carvalho de Almeida Prado**

\footnotetext{
" Doutora em Linguística com estágio de Pós-Doutoramento pela Universidade de São Paulo e Supervisora do Setor de Capacitação em Inglês Aeronáutico do Instituto de Controle do Espaço Aéreo. E-mail: patricialucks@gmail.com

${ }^{* *}$ Doutora em Letras no programa Estudos Linguísticos e Literários em Inglês pela Universidade de São Paulo e Professora na School of Humanities da Fujian University of Technology (FJUT). E-mail: malilaprado@hotmail.com
} 
Resumo: Inglês aeronáutico é o termo empregado para se referir às comunicações que ocorrem entre pilotos e controladores de tráfego aéreo durante os voos em espaço aéreo internacional. Neste artigo, apresentaremos dados de quatro corpora de inglês aeronáutico: dois de comunicações aeronáuticas reais e dois de atividades didáticas envolvendo aprendizes brasileiros, sendo um de pilotos e o outro de controladores. Será discutida a metodologia empregada para a compilação de cada um deles e realizada uma análise contrastiva de termos oriundos dos quatro corpora. Os resultados poderão ser aplicados tanto para informar o desenvolvimento de currículos quanto na elaboração de atividades didáticas voltadas a esses aprendizes, a fim de que desempenhem suas atividades com segurança.

Palavras-chave: Corpus oral; Corpora de aprendizes; Inglês Aeronáutico; Inglês para Fins Específicos.

Abstract: Aeronautical English is the term used to refer to communications that take place between pilots and air traffic controllers during flights in international airspace. In this article, we present data from four corpora in English, two of real aeronautical communications, and two of Brazilian learners, being one of pilots and another of controllers. We discuss the methology employed to the compilation of the four corpora, and introduce a contrastive analysis of terms taken from the corpora. The results can be used both to inform or develop curricula and didactic activities aimed at these learners, so that they exercise their professions safely.

Keywords: Spoken Corpus; Learner corpora; Aeronautical English; English for Specific Purposes.

TradTerm, São Paulo, v.37, n. 1, janeiro/2021, p. 148-174

Número Especial - Linguística de Corpus www.revistas.usp.br/tradterm 


\section{Introdução}

A língua inglesa é, hoje, a lingua franca da aviação, utilizada nas mais diversas formas de comunicação entre os profissionais que atuam nesse contexto, sejam pilotos, controladores de tráfego aéreo, comissários de voo, mecânicos de aeronaves, entre outros. De acordo com a Organização de Aviação Civil Internacional $(\mathrm{OACl})$, aviation language é a linguagem utilizada pelos vários profissionais da aviação (ICAO 2010: 3.2.6). Por esse entendimento, é possível traduzir aviation English como "inglês para aviação". Porém, o termo aviation English pode ter outro sentido, mais específico, para o qual empregamos a tradução "inglês aeronáutico", categorizando uma sublinguagem especializada do inglês para aviação para "designar a comunicação aeronáutica por radiotelefonia, eminentemente, entre pilotos e controladores ou órgãos de comunicação aeronáutica, em solo ou no ar, como parte de um voo" (TosquILUCKS E SILVA 2020: 106). Essa comunicação apresenta características e peculiaridades que a diferenciam das outras, de modo que a $\mathrm{OACl}$ define "comunicação aeronáutica via radiotelefonia" como sendo o conjunto de interações trocadas por esses profissionais, caracterizada pelo uso da Fraseologia Padrão combinado com o uso de uma linguagem comum (plain language) que extrapola a Fraseologia, nos momentos em que esta não é suficiente. Assim, essa comunicação radiotelefônica em língua inglesa, que combina Fraseologia Padrão e plain English, é o que denominamos "inglês aeronáutico", um subconjunto do "inglês para aviação".

A Fraseologia Padrão é composta por um conjunto de frases e expressões pré-estabelecidas que tomam por base um vocabulário de aproximadamente 400 palavras do contexto da aviação, no qual palavras funcionais - como pronomes, verbos de ligação, verbos auxiliares e algumas preposições - costumam ser excluídas ou evitadas (ICAO 2004). 0 objetivo da Fraseologia Padrão é permitir a troca de informações de forma clara, concisa e precisa, em situações normais de voo (ICAO 2010), e é suficiente para lidar com a maioria das situações encontradas na prática diária do controle de tráfego aéreo. Porém, em situações inesperadas ou não usuais - que podem ter causas 
tão diversas quanto: condições meteorológicas adversas, emergências médicas, falhas no funcionamento da aeronave, problemas na pista ou no aeródromo, perigo aviário, suspeitas de atentado à segurança, entre tantas outras - essa linguagem pode se mostrar limitada para a comunicação. Nesses casos, é necessário recorrer à linguagem comum (plain language), que também deve ser restrita ao contexto aeronáutico e submetida aos mesmos padrões que caracterizam a Fraseologia. A fim de se certificar de que os pilotos e controladores que atuam em tráfego aéreo internacional estão aptos a empregar o inglês aeronáutico em suas comunicações, a $\mathrm{OACl}$ estabeleceu requisitos mínimos de proficiência a serem aferidos pelos órgãos reguladores dos países membros e recomendou práticas para a capacitação desses profissionais.

A fim de orientar o cumprimento dessas práticas, a $\mathrm{OACl}$ desenvolveu e publicou, em 2004, o Documento $9835^{1}$ - Manual de Implementação dos Requisitos de Proficiência Linguística da $\mathrm{OACl}$, com instruções gerais sobre os procedimentos em relação à capacitação e avaliação dos profissionais citados. Em relação à avaliação, esse documento apresenta uma Escala de Proficiência em Língua Inglesa em seis níveis progressivos (1 a 6) e estabelece o Nível 4 como nível mínimo operacional. As categorias linguísticas para avaliação são: estrutura, vocabulário, pronúncia, compreensão oral, fluência e interação.

0 Brasil, como país signatário da $\mathrm{OACl}$, tem buscado atender às recomendações estabelecidas no Doc 9835. Em nosso país, existem duas instituições responsáveis por implementar os requisitos de proficiência linguística. De um lado, a Agência Nacional de Aviação Civil (ANAC) regulamenta as ações relacionadas aos pilotos e, de outro lado, o Departamento de Controle do Espaço Aéreo (DECEA), órgão do Comando da Aeronáutica, que é o responsável pelos controladores de tráfego aéreo (TosQUILUCKS ET AL 2016).

Este artigo tem como objetivo apresentar resultados de duas pesquisas realizadas com quatro corpora, sendo dois compilados de comunicações

\footnotetext{
${ }^{1}$ No original: Manual on the Implementation of Language Proficiency Requirements. Este documento foi revisto e teve 2 a versão publicada em 2010.
}

TradTerm, São Paulo, v.37, n. 1, janeiro/2021, p. 148-174

Número Especial - Linguística de Corpus www.revistas.usp.br/tradterm 
radiotelefônicas e os dois outros de aprendizes brasileiros de inglês aeronáutico. Esse trabalho comparativo é parte de um projeto conjunto que tem como fim informar o desenvolvimento de currículos e a elaboração de materiais didáticos de inglês aeronáutico para estudantes brasileiros. Neste artigo serão apresentados alguns resultados de dados obtidos nas duas pesquisas, que são característicos do inglês produzido pelo aluno brasileiro, seja ele piloto ou controlador.

\section{A Linguística de Corpus e estudos voltados ao ensino de línguas}

Um corpus retrata como a língua é usada em determinados contextos, dependendo do objetivo estabelecido durante a coleta dos textos, podendo ser formado por textos escritos ou por transcrição da linguagem oral (TAGNIN 2013). Existem hoje, no mercado, diversos livros didáticos, dicionários e glossários baseados em corpora. Ano após ano, têm surgido novas pesquisas sobre os benefícios que os corpora podem proporcionar para o ensino de línguas estrangeiras, principalmente aquele denominado para fins específicos (LSP, em inglês Language for Specific Purposes) (vide FLOWERDEW 2004; O'KeEFFE, MCCARTHY E CARTER 2007).

Chapelle (2001: 34) usa o termo "corpus revolution" para descrever a possível influência da Linguística de Corpus no ensino de língua inglesa neste século. A autora afirma que estudos com base em corpus podem mudar radicalmente a forma como a gramática é ensinada a aprendizes de língua estrangeira. Dentre os benefícios para o aprendiz, Aston (2001: 03) elenca (i) maior conscientização em relação a como a língua é utilizada; (ii) maior autonomia em tomar decisões sobre como realizar seus estudos; e (iii) habilidade de avaliar mais criticamente as informações colhidas diretamente nas fontes.

Já em 2011, Viana e Tagnin (2011) constataram que a atividade na área de Linguística de Corpus vinha aumentado de forma consistente no Brasil, principalmente desde a década de 90 . No entanto, em uma reflexão proposta

TradTerm, São Paulo, v.37, n. 1, janeiro/2021, p. 148-174

Número Especial - Linguística de Corpus

www.revistas.usp.br/tradterm 
18 anos depois da previsão do "corpus revolution" em Chapelle (2001), Chambers (2019) afirma que ainda há poucas propostas de uso de corpora no ensino de idiomas, apesar de seus benefícios para a área serem muitos. A mesma limitação é verificada em Tosqui-Lucks (2018) em um estado da arte sobre corpora no campo do inglês aeronáutico, área em que se insere a investigação aqui proposta: se, por um lado, há cada vez mais corpora sendo compilados em diferentes partes do mundo, por outro, poucos são efetivamente aplicados ao ensino e à avaliação.

Para citar alguns dos benefícios que a Linguística de Corpus pode trazer, partimos da elaboração do conteúdo programático de um curso, que é uma lista sequencial de tópicos, situações, habilidades e maneiras de ensinar, ou seja, uma projeção do que o aluno irá aprender ao longo de um curso e que reflete os objetivos gerais e específicos que o professor pretende que seus alunos atinjam ao término do curso (ALMEIDA E PRADo 2011). A seleção dos itens que compõem o conteúdo foi, por muitos anos, feita de maneira intuitiva, de acordo com o que desenvolvedores de materiais julgavam ser mais necessário, e normalmente definida por meio de gradação de estruturas gramaticais e de vocabulário. Mais recentemente, com o advento do uso de ferramentas computacionais em estudos linguísticos, o uso de corpora no desenvolvimento de conteúdos programáticos em ESP começou a se mostrar um excelente recurso. Flowerdew (2009), por exemplo, isolou aproximadamente 1.000 termos que ocorreram mais de 10 vezes em um corpus de 100.000 palavras para desenvolver o conteúdo programático de um curso para biólogos. A autora, entretanto, alerta que a frequência de ocorrência de uma palavra, por si só, não indica, necessariamente, sua utilidade para fins de desenvolvimento de conteúdos programáticos. Assim, é recomendável que os materiais escolhidos para compor o corpus sejam relevantes para os aprendizes, apoiando-se na alta frequência de palavras ou expressões em uma variada gama de amostras de linguagem para se assegurar que os aprendizes estejam expostos à linguagem comumente utilizada.

No caso das comunicações entre pilotos e controladores, somente um corpus oral pode propiciar a descrição de como os profissionais realmente 
utilizam a língua no decurso de suas atividades. Corpora orais não são tão abundantes quanto corpora escritos devido a dificuldades de confecção, tais como acesso à conversa espontânea e a necessidade de autorização dos participantes. Transpostas tais barreiras, apresenta-se mais uma: a escolha de como melhor representar a língua falada. 0 modelo de transcrição escolhido deve respeitar elementos que estão além da produção verbal, tais como gestos, ações, entonação, tomada e sobreposição de turnos, entre outros (ADOLPHS E KNIGHT 2010). Entretanto, é grande a riqueza de dados que um corpus oral propicia ao pesquisador, principalmente quando se trata de um corpus específico, coletado conforme as restrições do gênero que se propõe investigar. Nesses moldes, um corpus pode revelar informações como: o comportamento de determinados termos naquela especialidade, fraseologias típicas daquela comunidade de prática (WENGER 1998), posição preferencial de certas palavras ou fraseologias no discurso, (FLOWERDEW 2004), entre outros.

O corpus pode ser diretamente acessado pelos aprendizes, por meio de uma abordagem denominada Data-driven learning (JoHNS 1991; Boulton 2017). Podem-se fornecer as linhas de concordância de determinado item, por exemplo, e pedir aos alunos que busquem os colocados ou, ainda, que tentem identificar padrões e inferir regras a partir dessas linhas. Também se pode propor a análise de textos com maior incidência de determinado elemento que se almeja abordar, solicitando aos alunos que busquem esse elemento autonomamente.

Outra abordagem bastante produtiva em termos pedagógicos é a análise de corpora de aprendizes (vide TAGNIN E FROMM 2011). Podemos definir corpora de aprendizes como "coleções eletrônicas de textos produzidos por aprendizes de segunda língua e/ou língua estrangeira, organizadas de acordo com critérios específicos" (GRANGER 2008: 25). Esses corpora possibilitam investigações em torno da interlíngua (vide SANTOS 2019) e seu desenvolvimento por meio da coleta diacrônica de textos (HOUSEN 2002), subuso e sobreuso de elementos na comparação entre o corpus de aprendizes e um corpus de usuários proficientes (DUTRA E SILERo 2010), e o emprego do corpus no desenvolvimento de atividades ou materiais didáticos (FLOWERDEW 1998).

TradTerm, São Paulo, v.37, n. 1, janeiro/2021, p. 148-174

Número Especial - Linguística de Corpus

www.revistas.usp.br/tradterm 
No caso de um corpus de aprendizes especializado, as restrições são ainda maiores. Alinhamo-nos com a posição de Sarmento (2009: 263), que afirma que, no caso de um corpus de tema muito específico, como na área de aviação, cabe ao pesquisador compilar os textos com base em um projeto de pesquisa específico em mente, tal como a produção de material didático. Não há limite para o tamanho do corpus ou grau de especialização envolvido, mas os parâmetros para limitar o tipo de texto, oral ou escrito, devem ser muito bem estabelecidos.

\section{Metodologia}

Este artigo toma como base duas pesquisas realizadas sob a orientação da Professora Stella Tagnin, uma de doutorado e outra de pós-doutorado. As investigações foram realizadas nos corpora descritos a seguir, por meio do software Wordsmith Tools (WST) (SCOTT 2016). Nossa abordagem de uso da Linguística de Corpus é tanto baseada ou informada pelo corpus quanto dirigida pelo corpus (TOGNINI-BonelLI 2001). É baseada pelo corpus uma vez que procuramos características linguísticas particulares já pré-estabelecidas nos corpora e analisamos os dados encontrados para verificar as ocorrências, frequências, concordâncias e informações relevantes para compreender os fenômenos (RAYSON 2008). Mas também é dirigida pelo corpus no sentido que observamos as palavras e os clusters (blocos de linguagem ou expressões multipalavras) mais frequentes gerados na ferramenta Wordlist. Para a presente pesquisa, utilizamos dados levantados em Prado (2019) e Tosqui-Lucks (2019), e os comparamos utilizando WST e os corpora descritos na próxima subseção.

\subsection{Corpora de estudo}

Os quatro corpora tratados nas duas pesquisas, apesar de serem similares, em grande parte, no que diz respeito ao modelo transcritório (CRESTI 2000), divergem quanto às finalidades e período de coleta. Dois são compilados em ambiente profissional, de comunicações radiotelefônicas entre pilotos e 
controladores; os outros dois são corpora de aprendizes, por meio de transcrições de aulas ou de transcrições de produções orais de alunos dispostas em ambiente online.

O primeiro corpus foi inicialmente compilado por Prado (2015) para sua pesquisa de mestrado, e ampliado em Prado (2019) para a pesquisa de doutorado ${ }^{2}$. Esse corpus, composto por comunicações radiotelefônicas entre pilotos e controladores, predominantemente coletado a partir de sítios eletrônicos como o liveatc.com, buscou privilegiar situações anormais que possibilitassem a investigação do plain English nesse contexto. Para contemplar uma variedade linguística maior, foram priorizadas situações em que há interação entre tráfego internacional, ou seja, a aeronave envolvida na comunicação é de nacionalidade diferente do território ou espaço aéreo em que está. Intitulado RadioTelephony Plain English Corpus (RTPEC) (PRADO E TOSQUI-LUCKS 2019), o corpus teve como objeto de estudo os padrões léxicogramaticais mais recorrentes nas comunicações radiotelefônicas analisadas, com o intuito de oferecer melhor respaldo a professores de inglês aeronáutico em relação a dois dos critérios da escala da $\mathrm{OACl}$ - estrutura e vocabulário (Prado 2015).

Tosqui-Lucks (2017) compilou um corpus a partir da coleta e transcrição de gravações das comunicações rotineiras e não rotineiras, em língua inglesa, entre pilotos e controladores de tráfego aéreo do Sistema de Controle do Espaço Aéreo Brasileiro (Corpus SISCEAB) tendo como base, portanto, as comunicações que acontecem no espaço aéreo brasileiro. ${ }^{3}$ As gravações foram coletadas em localidades com fluxo intenso de tráfego aéreo internacional, durante um período de aproximadamente um ano e meio, a fim de compor uma amostra suficientemente representativa das situações alvo em diferentes regiões do Brasil. Foram coletadas aproximadamente 20 horas de comunicações

\footnotetext{
2 Pesquisas de mestrado e doutorado orientadas pela Profa. Stella Tagnin.

${ }^{3}$ Essa pesquisa recebeu apoio do CNPq por meio da Chamada Universal MCTI/CNPq -14/2013, processo 486237/2013-3. Sua realização foi previamente autorizada pelo Comando da Aeronáutica; porém, os dados, no Brasil, assim como em diversos países, não são acessíveis ao público em geral, por questões de segurança nacional. Assim, os dados brutos da pesquisa são considerados sigilosos. Análises e resultados são apresentados, preservando o anonimato dos participantes e de suas localidades.
}

TradTerm, São Paulo, v.37, n. 1, janeiro/2021, p. 148-174

Número Especial - Linguística de Corpus

www.revistas.usp.br/tradterm 
entre pilotos e controladores dos Centros de Controle de Área (ACC), 20 horas de comunicações entre pilotos e controladores dos Controles de Aproximação (APP) e 20 horas de comunicações entre pilotos e controladores das Torres de Controle (TWR). Esses dois corpora de certa forma se complementam, uma vez que Prado atua no ensino de pilotos brasileiros e Tosqui-Lucks na capacitação de controladores de tráfego aéreo do mesmo país.

Já em sua tese de doutorado, Prado (2019) investigou a importância da Pragmática, também a partir do RTPEC, analisando outros dois critérios de avaliação presentes na escala de proficiência da $\mathrm{OACl}$ - fluência e interação com propostas de aplicação para ensino e avaliação. Para investigar a aplicabilidade desses dois critérios, foram gravadas sete aulas no decorrer de um ano para um grupo de três pilotos de linha aérea, todos com cerca de 30 anos de experiência (também em voos internacionais). Essas aulas foram transcritas, gerando um corpus de pilotos aprendizes de aproximadamente 70.000 palavras. Os estudos provenientes desse corpus, junto com o RTPEC, revelam a importância de um ensino que se volte à conscientização da Pragmática.

Em sua pesquisa de pós-doutoramento ${ }^{4}$, concluída em 2019, Tosqui-Lucks também compilou um corpus de aprendizes. O corpus de aprendizes foi formado a partir das avaliações do curso a distância "Go4it" - curso de inglês aeronáutico para controladores de tráfego aéreo que atuam nos cinco ACCs do Brasil, localizados em diferentes regiões do país. Em cada avaliação desse curso, o aluno deve enviar ao tutor um áudio, elaborado e gravado por ele mesmo, sobre um determinado tema estudado no módulo didático, acompanhado pelo respectivo script desse áudio. Assim, o corpus de controladores aprendizes é composto pelos textos que correspondem às transcrições das atividades orais avaliadas. Os alunos são 44 controladores de tráfego aéreo profissionais, brasileiros, militares da Força Aérea Brasileira (sargentos ou suboficiais), inscritos nas cinco turmas oferecidas no ano de 2015. Foram priorizados apenas os módulos didáticos que contemplam atividades

4 Pesquisa supervisionada pela Profa. Stella Tagnin, com apoio da Coordenação de Aperfeiçoamento de Pessoal de Nível Superior - Brasil (CAPES) - Código de Financiamento 001.

TradTerm, São Paulo, v.37, n. 1, janeiro/2021, p. 148-174

Número Especial - Linguística de Corpus

www.revistas.usp.br/tradterm 
avaliadas na forma de comunicação radiotelefônica entre piloto e controlador. Ao todo, o corpus de aprendizes é composto por 244 textos e 19.276 palavras (tokens), excluindo-se as palavras pilot, controller, "ATCO" ou as letras "P" e "C" utilizadas para designar os interlocutores nas comunicações. Apesar de serem quatro os corpora compilados, para este estudo, apresentamos dados majoritariamente retirados de dois corpora: RTPEC e o corpus de controladores aprendizes. Os outros dois corpora, de pilotos aprendizes e SISCEAB, foram usados para complementar algumas dessas análises.

\section{Discussão}

Passamos a apresentar uma discussão sobre as análises de alguns termos produzidos pelos alunos brasileiros e uma comparação com o uso desses termos nos corpora de comunicações radiotelefônicas. Vale ressaltar que, no corpus de controladores aprendizes, foram preservadas as palavras com grafia errada, no intuito de investigar o que pode ser um indício de problema de pronúncia (como em mantein), de vocabulário (como em airbone, airborn e airborne) ou ainda quando a escolha de palavras se mostra equivocada, como no caso de approximation por approach ou alternative por alternate, como explicaremos em seguida.

Essa decisão nos obrigou a analisar cuidadosamente as ocorrências para verificar se a mesma palavra estava ou não sendo escrita de maneiras diferentes. A fim de ilustração, para confirmar uma informação, a Fraseologia recomenda o uso da expressão Affirmative ou, preferencialmente, de sua forma contrata Affirm, para evitar a confusão com a palavra Negative em caso de ruídos ou bloqueios por sobreposição de frequência na comunicação. Ao verificar as ocorrências dessas duas palavras, encontramos variações gráficas no corpus de controladores aprendizes, tais como affirmer, afim e afirmative.

0 total de 49 ocorrências do lema affirm contrasta com dezenove ocorrências de $O K$ e seis de yes, demonstrando clara preferência dos alunos em seguir o que preconiza a Fraseologia. Já o RTPEC, uma vez que partiu da transcrição de conversas sobre problemas técnicos, demonstra o oposto: há

TradTerm, São Paulo, v.37, n. 1, janeiro/2021, p. 148-174

Número Especial - Linguística de Corpus

www.revistas.usp.br/tradterm 
maior ocorrência de expressões de afirmação, tais como yes, yeah e okay, em um total de 796 vezes, contra 163 de ocorrências de affirm ou affirmative. A conclusão é de que a frequência de elementos mais conversacionais, como yes, yeah e okay, compõe a transição entre a Fraseologia Aeronáutica e o plain English. No corpus de pilotos aprendizes, as ocorrências das palavras affirm ou affimative se referem a discussões sobre a Fraseologia Aeronáutica, e não ao seu uso em si, devido à característica do discurso produzido em sala de aula. Ainda sobre essa questão, é mister destacar o uso de Roger, expressão que denota entendimento e concordância com o interlocutor. Com 463 ocorrências no corpus de controladores aprendizes é a palavra de conteúdo mais frequente do corpus, atrás apenas de pilot, que aparece com muitas ocorrências juntamente com ATCO porque os alunos utilizam essas palavras para designar os turnos das falas nos diálogos. Nas investigações, notamos que esse sobreuso de roger está ligado ao fato de os brasileiros utilizarem o termo para afirmar algo, uso não previsto pelos documentos de Fraseologia Aeronáutica e que, portanto, deve ser incluído no currículo para trabalho pedagógico.

Outras análises contrastivas entre os corpora oferecem diversas oportunidades de trabalho pedagógico. Elencamos algumas possibilidades, partindo de termos e clusters.

a) alternate: para se referir a "aeródromo alternativo" em inglês, o termo correto é alternate, que ocorreu 10 vezes no corpus de aprendizes contra seis de alternative. Isso nos levou a observar as ocorrências de alternative. Confirmando nossas suspeitas, detectamos que as seis ocorrências de alternative se referiam a aeródromos:

Tabela 1 - Uso equivocado de alternative no corpus de controladores aprendizes.

Concordance

inform minimum fuel to proceed to my alternative salvador. Pt lgm recife center sug

tain present heading and inform your alternative, remain endurance and endurance

Pilot: AAL204, So we will proceed to alternative airdrome, Santarém ACC: Roger

TradTerm, São Paulo, v.37, n. 1, janeiro/2021, p. 148-174

Número Especial - Linguística de Corpus www.revistas.usp.br/tradterm 


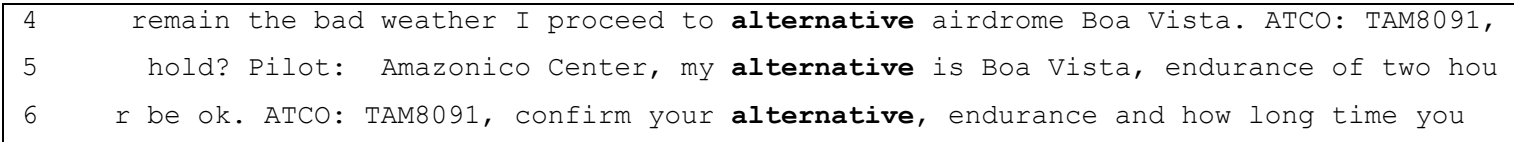

No RTPEC, a palavra alternate ocorre quatro vezes, enquanto o lema DIVERT (divert, diverting, diversion), correspondente ao verbo alternar ou ao substantivo alternado, ocorre quinze vezes. A preferência por alternate ou alternative pode dever-se à proximidade da palavra em português, alternativo, e foi selecionada para trabalho didático na comparação ao uso de diversion. Ressaltamos que o princípio desse trabalho não é eliminar o uso de alternate, previsto na Fraseologia Aeronáutica, mas ampliar a escolha lexical do aluno.

b) conditions: em nossa experiência na sala de aula, é comum que o aluno brasileiro, seja piloto ou controlador, use, equivocadamente, a expressão have condition/conditions para perguntar ou responder negativamente sobre ter capacidade de realizar alguma operação, quando o correto seria empregar to be able/unable, o que inclusive é o recomendado pela Fraseologia Padrão. Vejamos as ocorrências de condition do corpus de aprendizes para verificarmos nossa impressão nas aulas:

Tabela 2 - Ocorrências com condition no corpus de controladores aprendizes.

\begin{tabular}{|ll}
\hline $\mathrm{N}$ & Concordance \\
1 & speed. -N321XL 1.800 meters and the condition are good operation visual with wind \\
3 & rdrome and maceió operate on visual condition. Pt lgm request land in maceió. Pt 1 \\
4 & tence on the groud? TAP012 - I have condition and is necessary medical assistence \\
5 & er a have a passenger in a very bad condition, request divert to the nearest airpor \\
6 & ence on the groud ? TAP012 - I have condition and is necessary medical assistence \\
7 & ain FL200. And confirm, Do you have condition to proceed to GRU airport? Tam 3021 \\
8 & want back to guarulhos, we have not condition to proceed. AcC: GLO 8090, Center
\end{tabular}

Confirmando nossa observação como professoras, as linhas 4, 6, 7 e 8 são exemplos do uso inadequado da expressão have condition, em detrimento de be able / unable, previsto pela Fraseologia Aeronáutica. Observemos a forma plural, conditions, no mesmo corpus:

TradTerm, São Paulo, v.37, n. 1, janeiro/2021, p. 148-174

Número Especial - Linguística de Corpus www.revistas.usp.br/tradterm 
Tabela 3 - Ocorrências com conditions no corpus de controladores aprendizes.

\begin{tabular}{|lll}
\hline N & Concordance \\
1 & anopolis airport is bellow minima IFR conditions. Roger, so GLO1234 request to retu \\
2 & 8 TAM5434 CENTER: LAP778, do you have conditions to increase speed to Mach 0.80 for \\
3 & AAL222, Belem airport is below IFR conditions due to rain strong and all the run \\
4 & a information about meteorological conditions of salvador airdrome! Pilot: TAM \\
5 & but Recife now is operating under IFR conditions, do want to proceed? BAW245, aware \\
6 & W247 - Negative center, we can't have conditions to turn left. ATCO - BAW247,
\end{tabular}

As linhas 2 e 6 são exemplos que reforçam o uso inapropriado da expressão have condition utilizada no sentido de habilidade. Podemos abordar, em uma aula, a forma correta de empregar to be able por meio de exemplos do próprio corpus de controladores aprendizes, ou do RTPEC, como podemos observar:

Tabela 4 - Ocorrências com are you able no RTPEC.

\begin{tabular}{|ll|}
\hline $\mathrm{N}$ & Concordance \\
2 & orty-six thirty-three / are you able to do a couple of s tur \\
3 & nce one six nine five / are you able to vacate the runway? / \\
4 & ne zero four heavy / uh are you able to proceed or uh do you \\
5 & act uh maintain speed / are you able to do a hundred eighty \\
6 & e // LOT four one one / are you able to climb? // Confirm / \\
\hline
\end{tabular}

A expressão are you able to é utilizada para a constatação de manobrabilidade da aeronave. No estudo de outro cluster que poderia ser utilizado similarmente, can you, foi detectado que, das 82 ocorrências no RTPEC, somente em duas ocorreu o uso de verificação de manobras; as outras 80 ocorrências apresentaram uso pragmático, como ofertas ou solicitações.

Retomando o corpus de controladores aprendizes, há 36 ocorrências com formas declinadas de to be able no corpus, demonstrando que os alunos tendem a usar essa expressão corretamente. Porém, uma análise mais atenta mostra que nem sempre a construção está precisa. Observando a Tabela 6 com algumas dessas ocorrências, detectamos que muitas vezes os alunos deixam de usar to entre able e o verbo seguinte, como nas linhas 5, 7 e 10 a 11.

TradTerm, São Paulo, v.37, n. 1, janeiro/2021, p. 148-174

Número Especial - Linguística de Corpus www.revistas.usp.br/tradterm 
Tabela 5 - Linhas de concordância com able no corpus de controladores aprendizes.

\begin{tabular}{|c|c|}
\hline $\mathrm{N}$ & Concordance \\
\hline 1 & ilable Pilot: Amazonico Center, We aren't able to climb to FL450 and on FL 400 is imp \\
\hline 2 & 80. ATC: Negative, AAL239, FL390. Are you able?? PILOT: OK FL390. But I'm not able $n$ \\
\hline 3 & e you able?? PILOT: OK FL390. But I'm not able now just in 30 minutes. ATC: So repor \\
\hline 4 & 390 to avoid traffic?? PILOT: Affirm. I'm able to climb to FL380. ATC: Negative, AAL \\
\hline 5 & 11 provide priority for landing, are you able turn to the rigth for landing in Salv \\
\hline 6 & er. Descend to fl 310 and standby. Are you able to turn right to avoid traffic on des \\
\hline 7 & ber is 0.76. Atco Roger, tam 3301. Are you able increases you speed to mach .80 or hi \\
\hline 8 & 0 due to turbulence ATCO: TAM8051 are you able to reduce your speed due to traffic \\
\hline 9 & ction, fl210, A320. Pilot-Affirmer. I am able! Increase rate of descent to $3000 \mathrm{ft} /$ \\
\hline 1 & on localizer R18. Atco- Tam3301 Are you able increase rate of descent to $3000 \mathrm{ft} / \mathrm{mi}$ \\
\hline 11 & estimate on MCE VOR Pilot Tam33012 I am able increase speed to .81. Atco Roger, Ma \\
\hline 12 & on localizer R18. Atco Tam3301 Are you able increase rate of descent to $3000 \mathrm{ft} / \mathrm{mi}$ \\
\hline 13 & her is 0.76. Atco Roger Tam 3301. Are you able increases you speed to mach .80 or hi \\
\hline
\end{tabular}

Nossa recomendação é que o corpus seja levado para a sala de aula, e que os alunos sejam instigados a observar os usos e inferir as regras linguísticas. Após uma proposta de trabalho similar, observamos duas autocorreções no corpus de pilotos aprendizes:

Aluno 1: / I have condition <correção> I I I'm able to take off / /

Aluno 2: or after landing I I don't have <correção> I'm not able to vacate the runway //

Nesse corpus, houve quinze ocorrências da palavra able, contra duas da palavra condition(s). É importante que o professor trabalhe com as formas negativa e interrogativa e que atraia a atenção para quais situações tal emprego é recorrente, e porquê.

C) maintain: o estudo de Prado (2019) revelou que a palavra maintain pode causar confusão quando utilizada por brasileiros. Em situações em que pilotos brasileiros solicitaram a parada total da aeronave na pista de pousos e decolagens (interrompendo, assim, a operação daquela pista ou do próprio aeroporto), foi utilizada a expressão maintain on the runway, que foi compreendida como possibilidade de acidente. A palavra maintain, na

TradTerm, São Paulo, v.37, n. 1, janeiro/2021, p. 148-174

Número Especial - Linguística de Corpus www.revistas.usp.br/tradterm 
expressão 'maintain position', também causou mal entendidos em outras ocorrências reportadas. Verificamos no RTPEC que o termo maintain é normalmente utilizado com a aeronave em movimento, como em maintain speed ou maintain altitude, e que a expressão correta para indicar a parada da aeronave é hold position. Decidimos comparar esses dados com os do corpus de controladores aprendizes. Neste, é comum os ATCOs precisarem dar instruções com o verbo maintain, principalmente associada ao nível de voo $(\mathrm{FL}=$ flight level). Muitos alunos pronunciam esse verbo de forma incorreta ${ }^{5}$. Para agravar a situação, grande parte das vezes a instrução a ser dada ao piloto é climb and maintain, e o verbo climb muitas vezes também é pronunciado de forma errada, com o fonema /b/. Assim, uma recomendação seria propor uma atividade que praticasse a pronúncia dessa instrução. No corpus de controladores aprendizes, maintain apareceu 128 vezes, sendo, na maioria, climb and maintain. Menos frequente é a expressão descend and maintain, mas ela também apareceu no corpus.

Tabela 6 - Excerto com diversas ocorrências de climb and maintain no corpus de controladores aprendizes.

\begin{tabular}{|ll|}
\hline N & Concordance \\
1 & C: IBE6058, clear of traffic climb and maintain FL300. PILOT 3: Curitiba, DLH510, we \\
2 & roach control, squawk ident, climb and maintain FL300 PIL: glo2579, roger, climb to \\
3 & FL370? ATCO - Roger, AAL270, climb and maintain. PILOT - Recife center, Recife cente \\
4 & le increase speed to .81. Atco- Roger, Maintain.81. Inform Traffic above you leavin \\
5 & Recife center, squawk ident, climb and maintain fl300. Report reaching. Pilot- climb \\
6 & 22 f300. ATCO - DLH522, squak ident and maintain F300. PILOT - roger i'll.DLH522. ATC
\end{tabular}

0 cluster climb and maintain ocorre 53 vezes, enquanto descend and maintain ocorre dezesseis vezes no corpus de controladores aprendizes. Esse fato demonstra uma constância no uso correto da Fraseologia Aeronáutica, o que também nos possibilita afirmar que o domínio da fluência se dá na aquisição de clusters frequentemente utilizados, assim como previsto por Wood (2006) e Tagnin (2014).

\footnotetext{
${ }^{5} \mathrm{O}$ corpus transcrito é acompanhado das gravações realizadas pelos alunos, de modo que é possível analisar questões de pronúncia, entonação, prosódia etc.
}

TradTerm, São Paulo, v.37, n. 1, janeiro/2021, p. 148-174

Número Especial - Linguística de Corpus www.revistas.usp.br/tradterm 
Tabela 7 - Clusters com maintain no corpus de aprendizes

\begin{tabular}{|l|l|l|}
\hline 1 & CLIMB AND MAINTAIN & 53 \\
\hline 2 & MAINTAIN FLIGHT LEVEL & 27 \\
\hline 3 & AND MAINTAIN FLIGHT & 19 \\
\hline 4 & AND MAINTAIN FL300 & 17 \\
\hline 5 & DESCEND AND MAINTAIN & 16 \\
\hline
\end{tabular}

Observamos, dessa maneira, que é importante priorizar o ensino do uso da palavra maintain para os pilotos, e não para os ATCOs. Prado (2019) sugere uma sequência didática que promove esse trabalho para pilotos brasileiros.

d) right: Swinehart (2013) estudou, em um corpus do National Transportation Safety Board dos Estados Unidos, as ambiguidades polissêmicas do termo right, que pode significar "correto", "direita", "exatamente", "justamente", além de ser um marcador discursivo, um elemento de confirmação na elaboração de uma tag question, ou ainda um cumprimento ou um elemento de confirmação de uma situação (como sinônimo de alright). Como resultado, o autor verificou que apenas 18,2\% das ocorrências tinha o sentido previsto na fraseologia, ou seja, em quase $80 \%$ das ocorrências, o termo right é empregado de várias formas não padronizadas.

Encontramos nos corpora aqui compilados o uso de right com outras acepções além de "direita", única acepção desse termo também na Fraseologia Aeronáutica recomendada nos manuais da Força Aérea Brasileira. A Tabela 10, referente ao Corpus SISCEAB, confirma esse emprego na maioria das linhas de concordância. Entretanto, apresenta várias ocorrências da expressão temporal right now e também da expressão that's right: nas linhas 4, 5, 8, 10, 11 e 12 como confirmação e na linha 9 como um pedido de confirmação, de forma interrogativa.

Tabela 8 - Linhas de concordância com right no corpus SISCEAB

\begin{tabular}{|ll|}
\hline N & Concordance \\
1 & e, start (ininteligível) to make hold right now, turns to your left to... Lose \\
2 & n the direction to? ACC-CW Start hold right now. DTA741 Confirm, turn right,
\end{tabular}

TradTerm, São Paulo, v.37, n. 1, janeiro/2021, p. 148-174

Número Especial - Linguística de Corpus www.revistas.usp.br/tradterm 


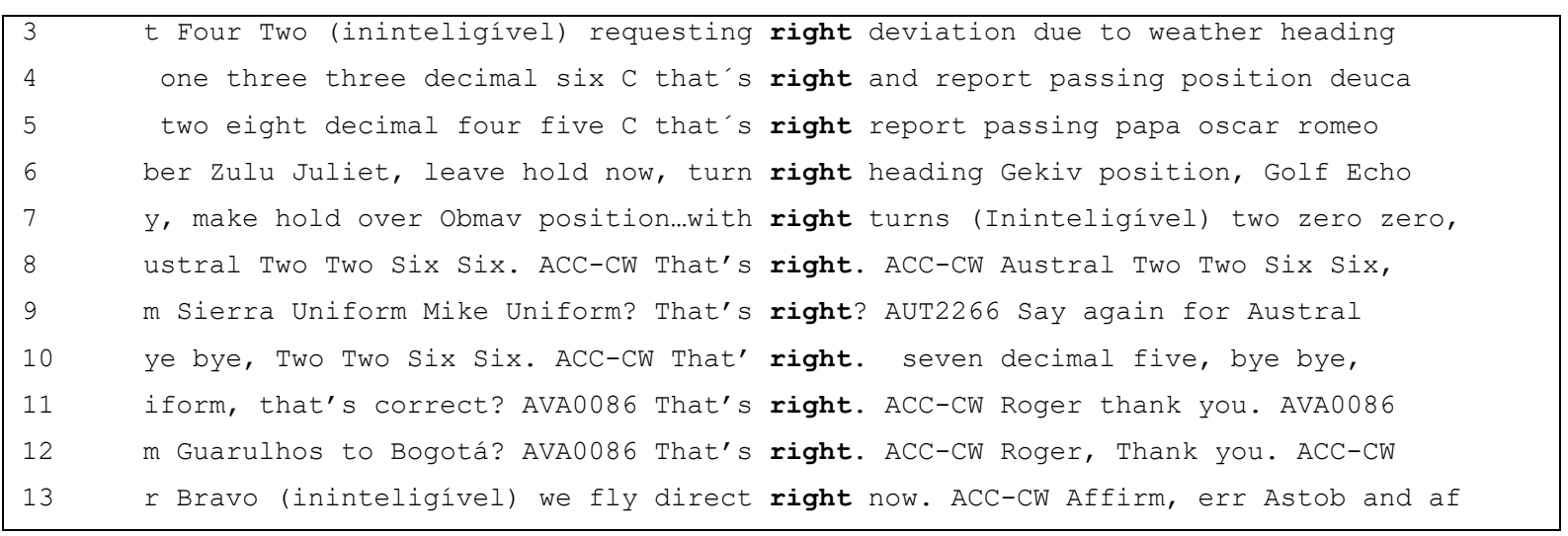

Já o corpus de controladores aprendizes traz 73 ocorrências de right, sendo sete com right now e duas com that's right. Na Tabela 11 temos as linhas de concordância de that's right no corpus de aprendizes, ambas usadas para expressar confirmação.

Tabela 9 - Linhas de concordância com that's right no corpus de controladores aprendizes

\begin{tabular}{|ll|}
\hline N & Concordance \\
1 & at FL 310 on opposite route. ACC - That's right. ACC: TAM3024 climb and \\
2 & present heading, GLO1234. GLO1234, that's right, you are number 2 on the
\end{tabular}

No RTPEC, o termo right foi evidenciado nas expressões that's right e all right, empregadas para abrir turnos (PRADO 2019). Também se revelaram úteis para indicar a transição da Fraseologia Aeronáutica para o plain English. Podese apresentar a importância do entorno da palavra right, nesse caso, that's e all, para realçar as funções que ela exerce no contexto radiotelefônico. Não se trata de julgar o uso de right como certo ou errado, mas de que o professor se certifique de que o aluno brasileiro reconheça e saiba empregar esse termo de usos tão versáteis corretamente.

e) clusters que representam a elaboração de hipóteses tais como it seems, it looks like e it appears to be: Prado (2019) apresenta uma discussão bastante relevante sobre como essas expressões são úteis para descrever algo de que se tem impressão no RTPEC. Já nos corpora compilados com os ATCOs, elas apresentam baixa frequência, apenas três ocorrências, como vemos nas

TradTerm, São Paulo, v.37, n. 1, janeiro/2021, p. 148-174

Número Especial - Linguística de Corpus www.revistas.usp.br/tradterm 
Tabelas 10 e 11. Isso indica uma possível necessidade de ensinar essas expressões, que se mostram úteis principalmente aos ATCOs quando necessitam descrever algum problema aparente na aeronave, para que os alunos passem a empregá-las. Vale relembrar que a característica principal do RTPEC é ter partido de problemas técnicos, podendo justificar o maior uso das expressões descritas acima.

Tabela 10 - Ocorrência de looks like no corpus de controladores aprendizes.

N Concordance

1 We have a passenger feeling bad, looks like he is having a heart attack.

Tabela 11 - Ocorrências de seems no corpus de controladores aprendizes.

$\mathrm{N}$ Concordance
1 to him a mask with oxygen, but it not seems to do effect. ACC-CW:
2 him entered to our aircraft, but now seems the problem is worse and he i

Já no corpus de pilotos aprendizes, tais expressões são um pouco mais recorrentes, particularmente em uma aula sobre descrição de figuras amparada pela demonstração de modelos de avaliações internacionais, como observamos:

Tabela 12 - Ocorrências de seems no corpus de pilotos aprendizes.

\begin{tabular}{|ll|}
\hline N & Concordance \\
2 & and close my eyes the world seems round around / became uh a \\
3 & dent B: she seems to be she seems to uuh not great/ to give \\
4 & C / P : this kind of answer seems for me to be confident // \\
5 & im // T: why not? // B: she seems to been more more smarter \\
& ng in ideas that uh she she seems to to be confident / she 1
\end{tabular}

Tabela 13 - Ocorrências de looks like no corpus de pilotos aprendizes.

\begin{tabular}{|ll|}
\hline $\mathrm{N}$ & Concordance \\
1 & to [stressed P: to to B: [looks like stressed P: kidding T: t \\
2 & e landscape the landscape look like B: beautiful // I'm plann \\
3 & B: a good time // and its look like a family and theyre prac \\
4 & inment / uh this this uh looks like a family // what do you \\
5 & English / but for me she looks like to have a better English
\end{tabular}

Observamos nessas linhas uma tentativa de produção do cluster exercendo a função de descrever aparências, porém sem o pleno domínio do

TradTerm, São Paulo, v.37, n. 1, janeiro/2021, p. 148-174

Número Especial - Linguística de Corpus

www.revistas.usp.br/tradterm 
seu uso gramatical (its look like, linha 3). Podemos utilizar essas linhas em um trabalho sequencial com os alunos e contrastá-las ao RTPEC, promovendo uma discussão sobre como as expressões são empregadas em relação à conjugação e ao entorno (adjetivos e substantivos).

f) so e just: Prado (2019) enfatiza a ocorrência de so e just no RTPEC como marcadores pragmáticos de iniciadores de turno, assim como as empressões that's right e all right. Confirmamos essa hipótese no corpus de controladores aprendizes no caso de so, como é possível observar na Tabela 14. Das ocorrências de so apresentadas na Tabela 14, apenas as linhas 1, 2, 3 e 10 não indicam início de turno; porém, podem indicar início do enunciado, com exceção da linha 3, em que so compõe a expressão thanks so much.

Tabela 14 - Ocorrências com so no corpus de controladores aprendizes.

\begin{tabular}{|c|c|}
\hline $\mathrm{N}$ & Concordance \\
\hline 1 & THE LEVEL OF FUEL IS NOT WORKING PROPERLY SO WE AREN'T ABLE TO INFORM EXACTLY OUR E \\
\hline 2 & that my fuel is not enough until RECIFE, so I request land in the nearest airdrome. \\
\hline 3 & Ilnot $1 \mathrm{~A}$ arrival. Recife airport, thanks so much. Pilot: Recife center, ONE6374. AT \\
\hline 4 & ilable, confirm intentions Pilot: AAL204, so we will proceed to alternative airdrome \\
\hline 5 & - Confirm your intentions. Piloto: Roger, so, I request divert to Galeao Airport an \\
\hline 6 & $r$ on board that needs medical assistance. So because this i resquest to alternate to \\
\hline 7 & y that you and another aircraft took off. So you can be without one tire. Do you und \\
\hline 8 & s very bad and the clouds are very heavy. So, I request divert to Londrina. Roger. D \\
\hline 9 & s Pilot: Roger, it's much heavy to climb. So, request FL380. Atco: SIA 706, descend \\
\hline 10 & r. We don't have fuel enough to make hold so request divert to Santos Dumont airport \\
\hline
\end{tabular}

O mesmo ocorre no corpus de pilotos aprendizes, em que as 319 ocorrências de so são majoritariamente no início de turnos. No caso de just, as ocorrências no corpus de controladores aprendizes são mais indicações temporais.

Tabela 15 - Ocorrências com just no corpus de controladores aprendizes.

\begin{tabular}{|ll}
\hline N & Concordance \\
1 & Negative, negative, I am gliding, I have just 20 minutes to fly. I will try make an \\
2 & ?? PILOT: OK FL390. But I'm not able now just in 30 minutes. ATC: So report ready to. \\
3 & passenger is a man, 40 years old and he just had a heart attack. Request ambulance \\
4 & the traffic in my 12 o'clock east bound, just confirm the level. PTCTP, the traffic
\end{tabular}

TradTerm, São Paulo, v.37, n. 1, janeiro/2021, p. 148-174

Número Especial - Linguística de Corpus www.revistas.usp.br/tradterm 
5 O: Roger, confirm the intentions. PILOT: just a moment... Recife.

PILOT: OK, Recife.

No corpus de pilotos aprendizes, just adquire a função de mitigador, atenuando a mensagem (CAFFI 1999), como em just a little, just a malfunction, entre outros. Também é comumente utilizado com expressões de tempo, assim como observado no corpus de controladores aprendizes.

g) expressões de polidez: a análise do RTPEC revelou alto uso de fórmulas pragmáticas, como thank you e thank you very much. Tal uso, assim como o emprego de outras fórmulas relacionadas à polidez, como cumprimentos, não é recomendado nos documentos de Fraseologia Aeronáutica. Ao contrastarmos esse uso no corpus de controladores aprendizes, agradecimento nas formas thanks ou thank you aparecem 20 vezes. Please ocorre 13 vezes. Vejamos as expressões com good:

Tabela 16 - Linhas de concordância com good

\begin{tabular}{|lll}
\hline N & Concordance \\
1 & -N321XL 1800 metres and the condition are good operation visual with wind 04 \\
2 & will try make an emergency landing. ATCo: Good luck sir, I'll let the rescuers. PI \\
3 & eport for descent. Crew : Curitiba Center, good evening, ARG1981, over MLO VoR, ma \\
4 & -ATCO: Negative, squalk code 3216 Teacher, good afternoon. Due I work on a rotation \\
5 & because the passenger's situation is not good. - ATCo: Roger, descend to FL100 \\
6 & doctor on board, but the situation is not good! We request to alternate aracaju \\
7 & n the ground, Because his situation is not good according to the doctor said. Cent \\
8 & ng. Passing FL145 to FL350. ACC-CW: AAL981, good morning, climb to FL190 due traffic \\
9 & approach control and the Fortaleza tower. Good Luck! BAw247 - Recife center, I re
\end{tabular}

Temos cinco ocorrências com good morning, uma com good afternoon, três com good evening e duas com good luck. Essas evidências corroboram outros estudos (LOPEZ 2013) que afirmam que, apesar de esses tipos de expressão não serem prescritos pela Fraseologia Aeronáutica, eles se tornam mais frequentes nos momentos em que há situações que fogem à rotina e que exigem maior negociação entre as partes.

TradTerm, São Paulo, v.37, n. 1, janeiro/2021, p. 148-174

Número Especial - Linguística de Corpus www.revistas.usp.br/tradterm 
As comparações realizadas neste artigo têm implicações pedagógicas relacionadas tanto às escolhas que devem pautar os currículos pedagógicos quanto ao insumo (input) que pode ser utilizado nas aulas. Nos currículos pedagógicos, ao considerarmos o uso que controladores e pilotos brasileiros fazem da língua inglesa e compararmos tal uso com outras possibilidades, damos-lhes a oportunidade de escolher formas linguísticas (CARTER E MCCARTHY 1997) e de conscientizarem-se do efeito que determinadas palavras ou expressões podem causar no interlocutor. Uma vez que o efeito no interlocutor é uma das preocupações da Pragmática Linguística (VERsChueren 2000), além da observação de elementos como as expressões de polidez, da função de iniciar turnos verificada em determinados clusters (como that's right e all right) e do uso de mitigadores (como just), acreditamos que as análises apresentadas aqui justificam um trabalho com a consciência pragmática (VERSCHUEREN 2000: 444), assim como previsto em Prado (2019). Sugerimos, portanto, uma abordagem data driven, que parta da reflexão sobre língua produzida oralmente, em contextos reais (PRADO E TOSQUI-LUCKS 2017), para então considerar as escolhas linguísticas feitas pelos falantes.

\section{Considerações Finais}

Ainda que os resultados levantados aqui não sejam exaustivos, pretendem ser um ponto de partida para um projeto maior, em que se consideram tanto o desenvolvimento de materiais didáticos para os profissionais - pilotos e controladores de tráfego aéreo brasileiros, como também a análise contrastiva de outros corpora compilados em outros espaços aéreos. Acreditamos que a investigação de outros corpora pode revelar aspectos que compreendem o uso de termos (como maintain), funções comunicativas (como iniciadores de turno), elementos pragmáticos (como expressões de polidez), entre outros.

Ainda que não tão abundantemente quanto desejável, é possível identificar que há um aumento de pesquisas com corpora no campo do inglês aeronáutico, da mesma forma que há especialistas afirmando que a Linguística

TradTerm, São Paulo, v.37, n. 1, janeiro/2021, p. 148-174

Número Especial - Linguística de Corpus

www.revistas.usp.br/tradterm 
de Corpus pode trazer contribuições significativas para a área, tanto para o ensino quanto para a elaboração de instrumentos de avaliação. Corroborando essa afirmação, atestamos, com entusiasmo, que a Linguística de Corpus é uma das três áreas de pesquisas realizadas pelos membros do Grupo de Pesquisa da ICAEA (International Civil Aviation English Association) ${ }^{6}$ criado em 2017. É também uma área produtiva dentro do GEIA (Grupo de Estudos em Inglês Aeronáutico $)^{7}$, grupos dos quais as autoras deste artigo fazem parte.

Para suprir a carência de materiais didáticos especialmente voltados ao público de pilotos e controladores de tráfego aéreo brasileiros, os resultados diretos desta pesquisa são aplicados em trabalhos como: elaboração de materiais didáticos para pilotos e controladores de tráfego aéreo; treinamentos operacionais, de manutenção e aperfeiçoamento, estágios etc.; proposta de revisão da Fraseologia Aeronáutica utilizada em situações não rotineiras e de emergência, bem como aperfeiçoamento dos manuais sobre esse assunto; reuniões e fóruns da ICAEA, que prestam assessoria direta à própria $\mathrm{OACl}$ em relação a sugestões para aprimoramento dos documentos que regulam as ações de capacitação e avaliação de pilotos e controladores em todos os estados membros; outras aplicações indiretas que poderão contribuir para a prevenção de incidentes e acidentes, como palestras, ações de conscientização, entre outras.

Os corpora aqui apresentados podem ser ampliados continuamente. Longe de esgotar o assunto, as análises e atividades aqui apresentadas têm como objetivo oferecer um modelo que poderá ser seguido na elaboração de outros tipos de atividades, utilizando a consulta aos quatro corpora, buscando, principalmente, a importância de se considerararem os dois profissionais no uso real do inglês aeronáutico, tanto em momentos autênticos de uso profissional quanto em situações de aprendizagem.

\footnotetext{
${ }^{6}$ https://www.icaea.aero/about/icaea-research-group/

${ }^{7}$ https://geia.icea.gov.br/geia/inicial.php
} 


\section{Referências}

ADOLPHS, S.; KNIGHT, D. Building a spoken corpus: what are the basics? In: O'KEefFE, A.; MCCARTHY, M. The Routledge Handbook of Corpus Linguistics. London and New York: Routledge, 2010: 38-52.

AlmeIDA, D.; Prado, M. Desenvolvendo o conteúdo programático de um curso de inglês para mecânicos de aeronaves com base em um corpus DIY: um estudo de caso. Aviation in Focus, v. 2, n. 2, Porto Alegre (PUC-RS), ago./dez. 2011, pp. 6-20.

Aston, G. Learning with Corpora. Bolonha: Athelstan, 2001.

Boulton, A. Data-driven learning and language pedagogy. In: THORNE, S.; MAY, S. Language, Education and Technology: Encyclopedia of Language and Education. New York: Springer, 2017.

CAfFI, C. On mitigation. Journal of Pragmatics, v. 31, 1999, pp. 881-909.

CaRTER, R.; MCCARTHY, M. Exploring Spoken English. Cambridge: Cambridge University Press, 1997.

Chambers, A. Towards the corpus revolution? Bridging the research-practice gap. Language Teaching, Cambridge, v. 52, n. 4, October 2019, pp. 460-475.

Chapelle, C. A. Computer Applications in Second Language Acquisition. Foundations for teaching, testing and research. Cambridge: Cambridge Applied Linguistics Series, CUP, 2001.

Cresti, E. Corpus di italiano parlato. Firenze: Accademia della Crusca, v. 1, 2000.

DUtRA, D. P.; Silero, R. P. Descobertas linguísticas para pesquisadores e aprendizes: a linguística de Corpus e o ensino de gramática. Revista Brasileira de Linguística Aplicada, v. 10, n. 4, Belo Horizonte, 2010, p. versão online. Disponivel em:

<http://www.scielo.br/scielo.php?script=sci_arttext\&pid=S198463982010000400005>. Acesso em: 25 mar. 2019.

FLOWERDEW, L. Corpus linguistic techniques applied to textlinguistics. System, v. 26, 1998, pp. 541-552.

FLOWERDEW, L. The argument for using English specialized corpora to understand academic and professional language. In: CONNOR, U.; UPTON, T. A. Discourse in the Professions: Perspectives from corpus linguistics. Amsterdam/Philadelphia: John Benjamins Publishing Company, 2004: $11-36$.

TradTerm, São Paulo, v.37, n. 1, janeiro/2021, p. 148-174

Número Especial - Linguística de Corpus www.revistas.usp.br/tradterm 
FLOWERDEW, L. Applying corpus linguistics to pedagogy - A critical evaluation. International Journal of Corpus Linguistics, v. 14, n. 3, 2009, pp. 393417.

GRANGER, S. A contribuição de corpora de aprendizes às áreas de aquisição de segunda língua e ensino de língua estrangeira: uma avaliação crítica. In: AIJMER, K. Corpora and Language Teaching. Tradução de Malila Carvalho de Almeida Prado e Armando Silveiro. Amsterdam: Benjamins, 2008.

HOUSEN, A. A corpus-based study of the L2-acquisition of the English verb system. In: Granger, S.; Hung, J.; PetCh-Tyson, S. Computer Learner Corpora, Second Language Acquisition and Foreign Language Teaching. Amsterdam; Philadelphia: John Benjamins, 2002: 77-116.

International Civil AVIation Organization. (ICAO) Manual on the Implementation of ICAO Language Proficiency Requirements. Doc 9835. $1^{\text {st }}$ edition. Montreal: ICAO, 2004.

International CIVIL AVIATION Organization. (ICAO) Manual on the Implementation of ICAO Language Proficiency Requirements. Doc 9835. $2^{\text {nd }}$ edition. Montreal: ICAO, 2010.

JOHNS, T. Should you be persuaded: Two samples of data-driven learning materials. English Language Research Journal, v. 4, 1991, pp. 1-16.

LOPEZ, S. Norme(s) et usage(s) langagiers: le cas des communications pilotecontrôleur en anglais. Linguistique.Université Toulouse le Mirail Toulouse II, 2013.

O'KeEFFE, A.; MCCARTHY, M.; CARTER, R. From corpus to classroom: language use and language teaching. Cambridge: CUP, 2007.

PrADO, M. Levantamento dos padrões léxico-gramaticais do inglês para aviação: um estudo vetorado pela Linguística de Corpus. São Paulo: Faculdade de Filosofia, Letras e Ciências Humanas, Universidade de São Paulo, 2015.

Prado, M. A relevância da Pragmática no ensino do inglês aeronáutico: um estudo baseado em corpora. São Paulo: Faculdade de Filosofia, Letras e Ciências Humanas, Universidade de São Paulo, 2019.

Prado, M.; TOSQUI-LUCKS, P. Are the LPRs focusing on real life communications issues? Internation Civil Aviation English Association. Dubrovnik: EmbryRiddle Scholarly Commons, 2017, pp. 1-20.

Prado, M.; TosquI-LuCKS, P. Designing the Radiotelephony Plain English Corpus (RTPEC): A specialized spoken English language corpus towards a description of aeronautical communications in non-routine situations. Research in Corpus Linguistics, v. 7, 2019, pp. 113-128.

TradTerm, São Paulo, v.37, n. 1, janeiro/2021, p. 148-174

Número Especial - Linguística de Corpus www.revistas.usp.br/tradterm 
RAYSON, P. From key words to key semantic domains. International Journal of Corpus Linguistics, v. 13, n.4, 2008, pp. 519-549.

SANTOS, G. Second language pragmatics: a corpus-based study of the pragmatic marker like. Letrônica: Revista Digital do Programa de Pós-Graduação em Letras da PUCRS, v. 12, n. 3, Porto Alegre (PUC-RS), dez 2019, p. e34002. Disponível em < http://revistaseletronicas.pucrs.br/ojs/index. php/letronica/article/view/34002>. Acesso em 02 mar 2020.

SARMENTO, S. Linguística de Corpus e o desenvolvimento de material didático para inglês. In: SARMENTO, S.; FreitAS, A. L. $O$ ensino do inglês como língua estrangeira: Estudos e Reflexões II. Porto Alegre: EDIPUCRS, 2009.

ScoTt, M. WordSmith Tools. Versão 7.0. Liverpool: Lexical Analysis Software, 2016.

SWINEHART, N. Aviation English Corpus Linguistics: Using the Right Phraseology?. Aviation English Corpus Linguistics, 2-5, 2013.

TAGNIN, S. O jeito que a gente diz: expressões convencionais e idiomáticas. Edição revista e ampliada. São Paulo: Disal, 2013.

TAGNIN, S. Using Corpus Linguistics to Overcome the Language Barrier. In: SCHUSTER, E.; LeVkOWITZ, H.; OliVEIRA, O. Writing scientific papers in English successfully: your complete roadmap. Andover e São Carlos: hyprtek, 2014, pp. 84-114.

TAGNIN, S.; Fromm, G. CoMAprend - a experiência da construção de um corpus de aprendizes para estudos. Domínios de Lingu@gem, v. 2, n. 2, 2011. Acesso em 29 de maio de 2020. Disponível em http://www.seer.ufu.br/index.php/dominiosdelinguagem/article/view/ $11493 / 6770$

Tognini-Bonelli, E. Corpus Linguistics at Work. Amsterdam: John Benjamins, 2001.

TosQUI-LuCKS, P. Compilação de um corpus de comunicações radiotelefônicas em língua inglesa do SISCEAB. Relatório Final de Pesquisa Tecnológica. Brasília: Conselho Nacional de Desenvolvimento Científico e Tecnológico, 2017, 126 p.

Tosqui-LuCKS, P. Aplicações de corpora no ensino e na avaliação de inglês aeronáutico: estado da arte, reflexões, direcionamentos. In: SCARAMUCCI, M.; TosquI-LuCKS, P.; Damião, S. Pesquisas sobre Inglês Aeronáutico no Brasil . Campinas, 2018, pp. 89-111.

TOSQUI-LUCKS, P. Elaboração de um módulo de atividades didáticas a partir de corpora de comunicações piloto-controlador em língua

TradTerm, São Paulo, v.37, n. 1, janeiro/2021, p. 148-174

Número Especial - Linguística de Corpus

www.revistas.usp.br/tradterm 
inglesa. Relatório Final de Pesquisa de Pós-Doutoramento. São Paulo: Faculdade de Filosofia, Letras e Ciências Humanas, Universidade de São Paulo, 2019, 136 p.

Tosqui-Lucks, P.; Souza, P. R.; RaYmundo, N.; GuerReiro, N.; ARAgão, B. F. Ensino e Avaliação de Língua Inglesa para Controladores de Tráfego Aéreo como Requisito de Segurança em Voo. Revista Conexão Sipaer, v. 7, n. 1, 2016, pp. 44-54. Disponível em <http://pesquisa.icea.gov.br/geia/publicacoes.php>. Acesso em $08 \mathrm{de}$ janeiro de 2018.

TosquI-LuckS, P.; SILVA, A. L. B. C. 2020. Da elaboração de um glossário colaborativo à discussão sobre os termos inglês para aviação e inglês aeronáutico. Estudos Linguísticos, São Paulo, v. 48. 4, p. 97-116. Disponível em <https://revistas.gel.org.br/estudoslinguisticos/issue/view/66/showToc>. Acsso em 2 Jun. 2020.

VERSCHUEREN, J. Notes on the role of metapragmatic awareness in language use. Pragmatics, v. 10, n. 4, 2000, pp. 439-456.

VIANA, V.; TAGnin, S. Corpora no ensino de línguas estrangeiras. São Paulo: Hub Editorial, 2011.

WENGER, E. Communities of practice: Learning, meaning, and identity. Cambridge: Cambridge University Press, 1998.

WoOD, D. Uses and functions of formulaic sequences in second language speech: An exploration of the foundations of fluency. Canadian Modern Language Review, v. 63, n. 1, 2006, pp. 13-33.

Recebido em: 04/05/2020

Aceito em: 05/10/2020

Publicado em janeiro de 2021

TradTerm, São Paulo, v.37, n. 1, janeiro/2021, p. 148-174

Número Especial - Linguística de Corpus www.revistas.usp.br/tradterm 